\title{
Platelet-Rich Plasma Supports Proliferation and Redifferentiation of Chondrocytes during In Vitro Expansion
}

\author{
Vivek Jeyakumar ${ }^{1 *}$, Eugenia Niculescu-Morzsa ${ }^{1}$, Christoph Bauer ${ }^{1}, Z_{\text {Zsombor Lacza }}^{2}$ \\ and Stefan Nehrer ${ }^{1}$
}

${ }^{1}$ Centre for Regenerative Medicine and Orthopedics, Danube University Krems, Krems an der Donau, Austria, ${ }^{2}$ OrthoSera $\mathrm{GmbH}$, Krems an der Donau, Austria

Articular cartilage regeneration is insufficient to restore sports injuries or defects that can occur from trauma. Treatment options for cartilage repair include autologous chondrocyte implantation (ACl) by isolation, expansion, and reimplantation of healthy donor chondrocytes. Chondrocyte expansion onto 2D substrates leads to dedifferentiation and loss of the cellular phenotype. We aimed to overcome the state of dedifferentiation

OPEN ACCESS

Edited by: Maddalena Mastrogiacomo, Università di Genova, Italy

Reviewed by: Chiara Gentili,

Università di Genova, Italy

Christian Albrecht,

Medical University of Vienna, Austria

Mona Kamal Marei,

Alexandria University, Egypt

*Correspondence:

Vivek Jeyakumar vivek.jeyakumar@donau-uni.ac.at

Specialty section: This article was submitted to

Tissue Engineering and

Regenerative Medicine,

a section of the journal Frontiers in Bioengineering and Biotechnology

Received: 05 September 2017 Accepted: 23 November 2017 Published: 06 December 2017

Citation: Jeyakumar V, Niculescu-Morzsa E, Bauer C, Lacza Z and Nehrer S (2017) Platelet-Rich Plasma

Supports Proliferation and Redifferentiation of Chondrocytes during In Vitro Expansion. Front. Bioeng. Biotechnol. 5:75. doi: 10.3389/fbioe.2017.00075 by biochemical stimuli with platelet derivatives such as platelet-rich plasma (PRP) and hyperacute serum (HAS) to achieve sufficient cell numbers in combination with variable oxygen tension. Human articular chondrocytes from osteoarthritic (OA) cartilage chondrocytes were switched from 10\% FCS supplementation to either 10\% PRP or $10 \%$ HAS after initial passaging for further experiments under normoxic $\left(20 \% \mathrm{O}_{2}\right)$ or hypoxic $\left(1 \% \mathrm{O}_{2}\right)$ conditions. An XTT assay measured the effect of PRP or HAS on the cell proliferation at 3, 6, and 9 days. The chondrogenic redifferentiation potential of dedifferentiated chondrocytes was determined with reverse transcriptase quantitative real-time PCR for markers of expression for type II collagen (COL2A1), type I collagen (COL1A1), and matrix metalloproteinases MMP3, matrix metalloproteinase 13 (MMP13) at 24 and $72 \mathrm{~h}$. Measured protein levels of 100\% PRP or HAS by multiplex quantification revealed basic fibroblast growth factor, G-CSF, and PDGF were significantly higher in PRP than in HAS $(p<0.05)$ but LEPTIN levels did not differ. The quantified protein levels did not differ when isolated from same donors at a different time. Chondrocyte proliferation indicated that supplementation of $10 \%$ HAS enhanced the proliferation rate compared to $10 \%$ PRP or $10 \%$ FCS at 6 and 9 days significantly $(p<0.05)$. mRNA levels for expression of COL1A1 were significantly downregulated $(p<0.05)$ when cultured with $10 \%$ PRP than $10 \%$ HAS or 10\% FCS under normoxic/hypoxic conditions. COL2A1 was significantly upregulated $(p<0.05)$ in PRP than 10\% HAS or 10\% FCS. MMP3 expression was downregulated after $72 \mathrm{~h}$ under all conditions. MMP13 was upregulated with 10\% PRP at both 24 and $72 \mathrm{~h}$ but significantly downregulated under hypoxia $\left(1 \% \mathrm{O}_{2}\right)$ for all circumstances. While HAS has its effect on chondrocyte proliferation, PRP enhances both proliferation and redifferentiation of dedifferentiated chondrocytes. PRP can replace standard usage of FCS for chondrogenic priming and expansion as implications for clinical use such as $\mathrm{ACl}$ procedures.

Keywords: chondrocytes, dedifferentiation, autologous chondrocyte implantation, platelet-rich plasma, hyperacute serum 


\section{INTRODUCTION}

Articular cartilage is an avascular, alymphatic, aneural tissue that functions as a lubricating and load-bearing surface of the joint limited by the regenerative potential of its individual cell type, the chondrocyte. A defect at the site of tissue can often lead to larger lesions and without prompt treatment can result in a degenerative disorder such as osteoarthritis (OA). Autologous chondrocyte implantation (ACI) predominantly treats focal chondral defects $\geq 3-4 \mathrm{~cm}^{2}$ covered by periosteum layer (ACI-P) (Minas, 2001), collagen type I/III membrane (ACI-C) (Niemeyer et al., 2008), or the use of fibrin or hyaluronan matrix-assisted chondrocyte implantation (ACI-M/MACI) (Nehrer et al., 2008). ACI is a technique that involves isolation of autologous articular chondrocytes from a non-load-bearing site of the joint by enzymatic digestion, expanded, and implanted into the defect site (Peterson et al., 2000). Isolation of chondrocytes from the tissue biopsy involves enzymatic digestion which further dissociates the chondrocytes from their pericellular microenvironment (chondrons) that dedifferentiates the cells by losing their native chondrogenic phenotype (Vonk et al., 2014). Despite tremendous clinical success with ACI, there still exist areas for continued development of efficient protocols toward priming hyaline cartilage formation.

During in vitro expansion for ACI, articular chondrocytes are inclined to dedifferentiate toward a fibroblast-like phenotype by reducing expression of collagen type II (COL2A1) and increasing expression of collagen type I (COL1A1) at both the mRNA and protein levels (Schnabel et al., 2002). The dedifferentiation process has been indicated to progress over several passages in monolayer culture limiting the opportunity to obtain surmountable quantities of cells for transplantation in a differentiated state (Kang et al., 2007). The process of dedifferentiation can be overcome by supplementing culture medium with exogenous growth factors (Stewart et al., 2000; Pei et al., 2002) or culturing in biomaterials such as alginate or collagen type I and/or type II (hyaluronan) (Nehrer et al., 1997; Stevens et al., 2004; Mesenchymal, 2009). Redifferentiation of chondrocytes in vitro requires a multifaceted approach utilizing biochemical stimuli involving recombinant growth factors such as transforming growth factor- $\beta$, insulin-like growth factor-1, fibroblast growth factor-2, and platelet-derived growth factor BB (PDGF-BB) (Martin et al., 1999; Brandl et al., 2010). Biophysical stimuli include oxygen tension or mechanical loading (Kawanishi et al., 2007; Duval et al., 2009). Chondrocytes cultured on monolayers under low-oxygen tension maintains an oxidative phenotype (Heywood and Lee, 2010) and post-expansion when aggregated in 3D cultures begin to express elevated levels of collagen type II expression (Egli et al., 2008; Henderson et al., 2010). Hypoxic culture condition has been shown to enhance markers of redifferentiation, increased matrix formation concurrently inhibiting hypertrophic markers in both articular and osteoarthritic-derived chondrocytes (Markway et al., 2013). Disadvantages over growth factor supplementation include their high-cost utility and the technical challenges associated with biomaterials. Alternative substitutes such as platelet derivative products constitute a source of growth enriching factors which is also advantageous for its cost effective, large scale manufacturing with high standardization for cell culture.
Platelet-rich plasma (PRP) has gained wide attention in the field of regenerative medicine and cell-based therapies as a human alternative replacing fetal bovine serum for cell propagation. The $\alpha$-granules in platelets constitute a natural source of growth factors and cytokines. Platelets can be ruptured either by repeated freeze-thaw cycles or activated by the addition of bovine thrombin to release the growth factor, cytokines from their granules referred in as platelet lysates (PL). The effects of both PRP and PL have been reported virtually by several in vitro studies on proliferation and differentiation of chondrocytes (Akeda et al., 2006; Drengk et al., 2009; Spreafico et al., 2009). In patients with knee joint degeneration, intra-articular injections of PRP injection of PRP seems to be a viable treatment for knee OA and has the potential to lead to symptomatic relief for up to 12 months (Smith, 2016). There appears to be an increased risk of local adverse reactions after multiple PRP injections but diminishes eventually (Filardo et al., 2015). Similarly, platelet-rich fibrin (PRF), a second-generation platelet derivative that can be formed by a natural coagulation process without any anticoagulants or biochemical modification (Dohan et al., 2006) is of interest for its hyperacute serum (HAS). HAS is separated from the blood cells during the PRF clotting phase within $10 \mathrm{~min}$, and this is why HAS contains only the cytokines which are released in a hyperacute phase of clot activation. Moreover, HAS is beneficial over PRP due to its relatively simple activation of platelets without the need for exogenous thrombin addition.

The technical requirement of ACI pose a paradoxical challenge that the harvested mature chondrocytes need to get dedifferentiated to proliferate, then, when a sufficient number of cells obtained, they need to redifferentiate in situ to form a new cartilage layer. Therefore, culture conditions of articular chondrocyte expansion in monolayers in vitro toward ACI must be standardized with biochemical stimuli to avoid the process of dedifferentiation and achieve sufficient expansion with minimal subculture. The goal of this study was to evaluate the potential of PRP and HAS, in combination with variable oxygen tension, to minimize chondrocyte dedifferentiation while maintaining adequate proliferation rates during in vitro monolayer expansion.

\section{MATERIALS AND METHODS}

\section{Human Subjects}

Human osteoarthritic cartilage was obtained from a total of six donors $(72.83 \pm 8.98$ years) undergoing total knee replacement surgery. Human blood was collected from 16 healthy male and female volunteers after written informed consent. The local ethical commission approved the study protocol (approval no. GS4-EK-4/249-2013).

\section{Preparation of Platelet Derivatives}

Whole blood was collected from 10 individual blood donors of $37.2 \pm 9.6$ years $($ mean $\pm \mathrm{SD}$ ) into 9-mL silicon-coated blood collection tubes (VACUETTE ${ }^{\circledR}$ z serum clot activator, Greiner bio-one) and centrifuged at $1,770 \mathrm{~g}$ for $10 \mathrm{~min}$. The resulting fibrin clot was removed from the tube, and a bottom portion containing the red blood cells was cut and discarded. The fibrin clot was then 
squeezed with a non-absorbable impermeable sterile material in a sterile Petri dish to extrude the HAS, and the HAS samples were stored at $-80^{\circ} \mathrm{C}$ until further use. PRP was prepared by transferring whole blood from the same donors into 9-mL EDTA-coated blood collection tubes (VACUETTE ${ }^{\circledR}$ K3EDTA, Greiner bio-one) and centrifuged at $440 \mathrm{~g}$ for $10 \mathrm{~min}$. The platelet enriched plasma (middle layer) along the platelet poor plasma (supernatant) was transferred into a $15-\mathrm{mL}$ falcon tube and centrifuged at $1,770 \mathrm{~g}$ for $10 \mathrm{~min}$. The resulting pellets that contained an average of $10^{7}$ platelets/mL were resuspended in the superficial plasma layer corresponding to the volume obtained from the HAS in these individual donors. The PRP was stored at $-80^{\circ} \mathrm{C}$ until further use.

\section{Growth Factor Evaluation}

The composition of growth factors in HAS and PRP respective to individual donor variation were quantitatively determined for multiple human protein biomarkers, performed by a bead-based multiplex analysis. Human biomarkers basic fibroblast growth factor (bFGF), leptin, PDGF-BB, and G-CSF (Bio-RAD, Vienna, Austria) were used for this assay, according to the manufacturer's instruction. Analyses of samples were performed with a BioPlex $^{\text {TM }} 200$ system (Bio-RAD, Hercules, CA, USA).

\section{OA Chondrocyte Isolation}

Cartilage tissues obtained from the six donors ( $72.83 \pm 8.98$ years) were washed in phosphate-buffered saline and minced. OA chondrocytes $(\mathrm{OAC})$ were isolated by enzymatic digestion as previously described (Jeyakumar et al., 2016) and expanded in growth medium (GIBCO ${ }^{\circledR}$ DMEM/F12 GlutaMAX ${ }^{\text {TM}}-$ I, Invitrogen, Vienna, Austria) containing $2.5 \mu \mathrm{g} / \mathrm{mL}$ Amphotericin B and $0.1 \mathrm{mg} / \mathrm{mL}$ streptomycin (Sigma, Steinheim, Germany) with either 10\% FCS (PAA Laboratories GmbH, Linz, Austria). All assays were performed on passage 1 chondrocytes to minimize the extent of dedifferentiation over subcultures.

\section{Cell Proliferation Assay}

OA chondrocytes were seeded onto the 96-well plates at a seeding density of 3,000 cells $/ \mathrm{cm}^{2}$ and cultured in control medium $(10 \%$ FCS) for $48 \mathrm{~h}$ for cells to adhere. Post 48 -h medium was replaced to treatment conditions with $10 \%$ HAS or $10 \%$ PRP or $10 \%$ FCS in experimental groups. Heparin $(2 \mathrm{U} / \mathrm{mL})$ was added to cultures with PRP to prevent gelation. The influence of HAS or PRP on cell proliferation was measured by the XTT (sodium $3^{\prime}$-[1(phenylamino carbonyl)-3,4-tetrazolium]-bis (4-methoxy-6nitro) benzene sulfonic acid hydrate) assay (Cell Proliferation Kit II; Roche Diagnostics GmbH, Mannheim, Germany) according to the manufacturer's instructions. Briefly, the XTT reagents were added to the cell culture medium and incubated for $4 \mathrm{~h}$ at $37^{\circ} \mathrm{C}$. Post-incubation, absorbance was measured at $450 \mathrm{~nm}$ with a background reference wavelength of $690 \mathrm{~nm}$ using a plate reader.

\section{Reverse Transcriptase Quantitative Real-time PCR (RT-qPCR)}

The transcriptional expression of chondrogenic-specific genes was investigated with a real-time quantitative reverse transcriptase polymer chain reaction (RT-qPCR), according to the manufacturer's instructions (Roche). Total mRNA was extracted using the high pure RNA isolation kit (Roche Diagnostics $\mathrm{GmbH}$, Mannheim, Germany). The mRNA was reverse transcripted with a first strand cDNA synthesis kit (Roche Diagnostics $\mathrm{GmbH}$, Mannheim, Germany), and cDNA samples were examined for chondrogenic markers COL1A1, COL2A1, MMP3, and matrix metalloproteinase 13 (MMP13) with Random Primer $\mathrm{p}(\mathrm{dN})_{6}$ according to the manufacturer's instruction with RT-qPCR analysis performed in a cycler. The amount of amplified endogenous GAPDH expression level was used as an external reference gene. A $2^{-\Delta \Delta C t}$ method was used to evaluate the relative expression level of mRNA for each target gene (Livak and Schmittgen, 2001) (Table 1).

\section{Statistical Analysis}

Non-parametric Mann-Whitney $U$-test were used to make comparisons between paired data, and multiple comparisons were performed by non-parametric Friedman tests followed by Dunn's post hoc test. All data are presented as the mean \pm SD. Statistical significance was set at $p<0.05$. All statistical analysis was performed using GraphPad Prism Software (Graphpad Prism Software Inc., San Diego, CA, USA).

\section{RESULTS}

\section{Determined Protein Levels in Individual HAS, PRP Donors, and Isolation Time Dissimilarities in Different PRP and HAS Donors}

Measured protein levels quantified from 10 individual blood donors revealed on average that bFGF was higher (Figure 1A) in PRP $(20 \mathrm{ng} / \mathrm{mL})$ than in HAS $(0.18 \mathrm{ng} / \mathrm{mL})(p<0.0001)$. G-CSF was higher (Figure 1B) in PRP $(17.6 \mathrm{ng} / \mathrm{mL})$ than in HAS $(0.61$ $\mathrm{ng} / \mathrm{mL})(p<0.0001)$. Leptin showed no significant differences (Figure 1C) between HAS (185.55 ng/mL) and PRP (126.73 $\mathrm{ng} / \mathrm{mL})(p=0.782)$. PDGFF-BB was higher (Figure 1D) in PRP $(582.66 \mathrm{ng} / \mathrm{mL})$ than in HAS $(89 \mathrm{ng} / \mathrm{mL})(p<0.0001)$. Similarly,

TABLE 1 | Sequences of primers and conditions used in reverse transcriptase quantitative real-time PCR.

\begin{tabular}{lll}
\hline Target gene & Primer forward & Primer reverse \\
\hline GAPDH & CTCTGCTCCTCCTGTTCGAC & ACGACCAAATCCGTTGACTC \\
COL2A1 & GTGTCAGGGCCAGGATGT & TCCCAGTGTCACAGACACAGAT \\
COL1A1 & GGGATCCCTGGACCTAAAG & GGAACACCTCGCTCTCCAG \\
$M M P 3$ & CAAAACATATTCTITGAGAGGACAA & TTCAGETATTCGCTGGGAAA \\
$M M P 13$ & TITCCTCCTGGGCCAAAT & GCAACAAGAACAAGTTGTAGCC
\end{tabular}




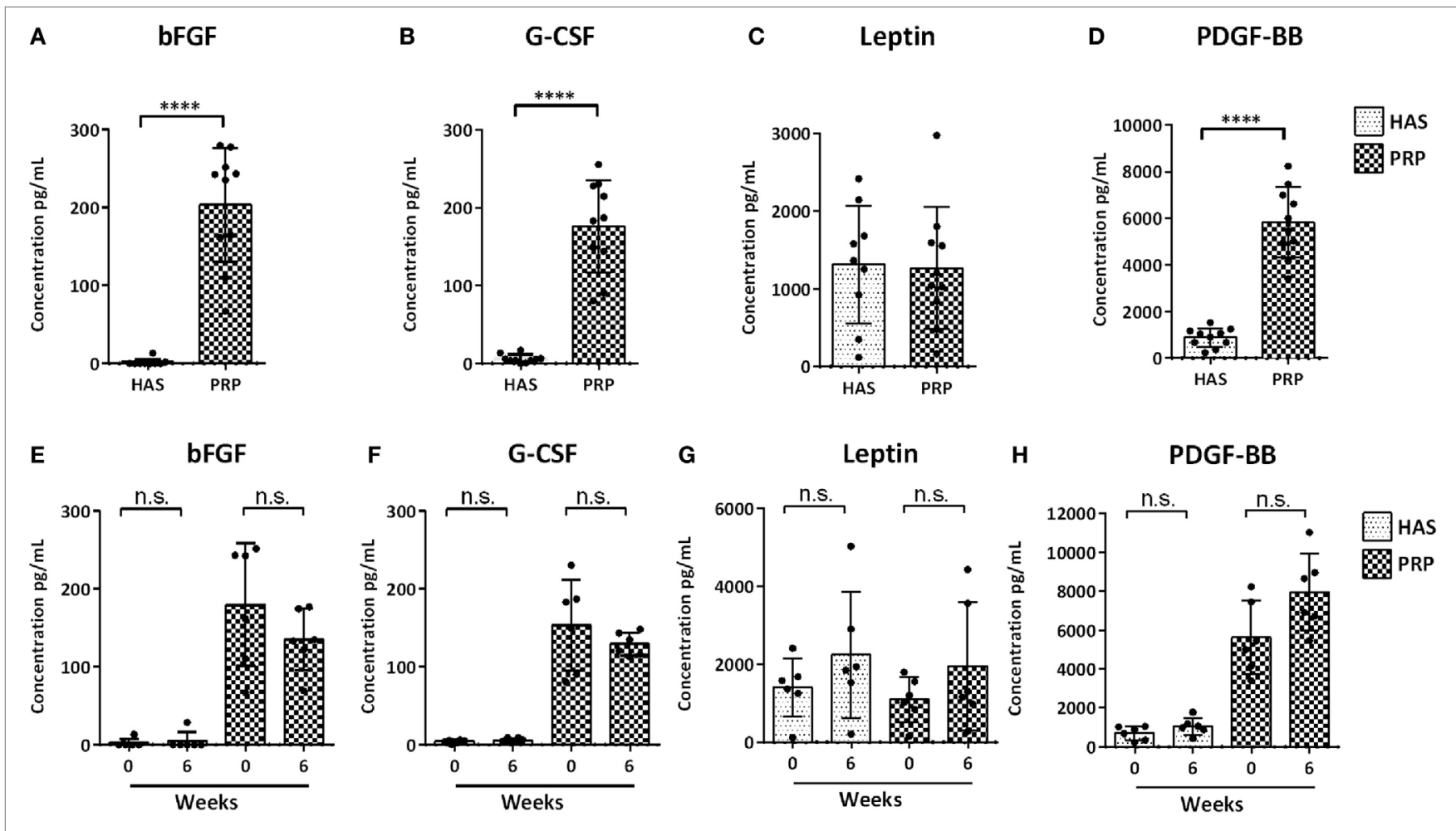

FIGURE 1 | Analysis of determined protein content of hyperacute serum (HAS) and platelet-rich plasma (PRP) from 10 individual blood donors (A-D) and differences in protein levels isolated from 6 blood donors at $\mathbf{O}$ and 6 weeks for determining differences in protein levels to different isolation time points (E-H) determined by a multiplex bead-based array. Data represented as mean \pm SD. Statistically significant is denoted by ${ }^{\star \star \star \star} p<0.0001$.

HAS and PRP isolated at 0 and 6 weeks from six individual donors revealed that isolation time dissimilarities in the release kinetics of PDGF-BB, G-CSF, leptin, and bFGF (Figures 1E-H) were not significantly different among the individual donors $(p=0.1797$, $p=0.4848, p=0.5043$, and $p=0.5887$, respectively). The proteins quantified were not dependent on isolation time for either the HAS or PRP preparations.

\section{HAS, PRP from Individual Blood Donors on OA Chondrocyte Proliferation}

Cell proliferation was determined based on the metabolic activity over 3, 6, and 9 days when cultured with 10\% FCS, 10\% PRP, or $10 \%$ HAS. No significant differences were observed over 3 days of culture among 10\% FCS versus $10 \% \operatorname{PRP}(p=0.5045)$ or $10 \%$ FCS versus $10 \%$ HAS ( $p=0.9425$ ). The metabolic activity, however, significantly increased at day 6 in $10 \%$ HAS versus $10 \%$ FCS $(p<0.0001)$ and 10\% PRP $(p<0.01)$. On day $9,10 \%$ HAS enhanced the metabolic activity even higher versus $10 \%$ FCS $(p<0.001)$ and $10 \%$ PRP $(p<0.01)$ (Figure 2). Metabolic activity of the cells remained almost the same at all time points between $10 \%$ FCS and 10\% PRP with no significant difference among them.

\section{Chondrogenic Gene Expression of OAC Cultured in Pooled HAS or PRP}

Quantitative RT-qPCR indicated that under normoxic conditions addition of 10\% PRP led to an increased differentiation index

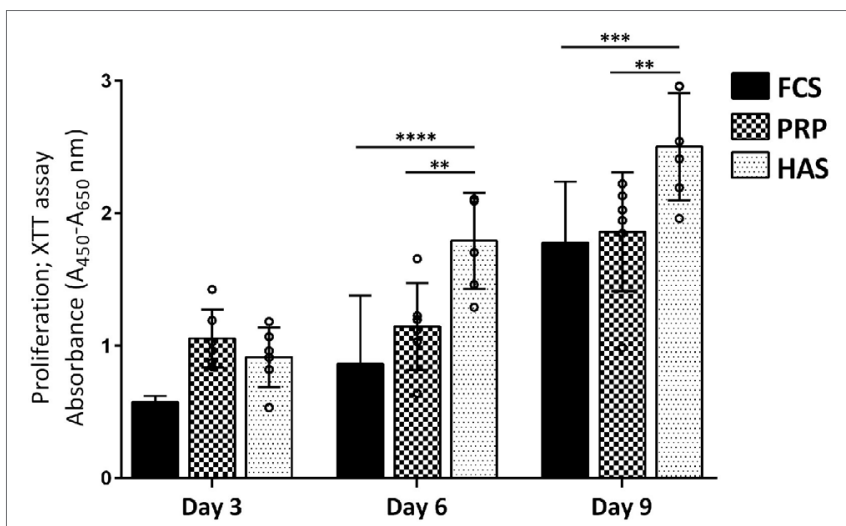

FIGURE 2 | Effect of platelet-rich plasma (PRP) or hyperacute serum (HAS) from six individual blood donors on the proliferation of $O A$ chondrocytes (OAC) from three donors. 10\% HAS stimulated a higher response from all three donors of OAC on day 6, 9 compared with $10 \%$ FCS or PRP. Statistically significant is denoted by ${ }^{* *} p<0.001$ and ${ }^{* * * *} p<0.0001 ; n=3$ biological replicates.

(COL2A1/COL1A1) from a dedifferentiated phenotype within 24 and 72 h. 10\% FCS led to a decrease in the index from 15 -folds at $24 \mathrm{~h}$ to $<1$-fold at $72 \mathrm{~h}$, and $10 \%$ HAS led to a decrease in the index both at 24 and $72 \mathrm{~h}$ compared to day 0 (Figure 3D). Under hypoxic conditions, the index for 10\% PRP increased from 24 to 72 h. From 24 to 72 h, 10\% FCS and 10\% HAS exhibited 
A

\section{COL1A1}

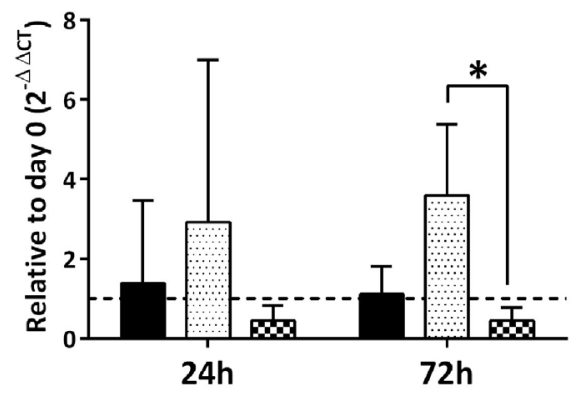

C

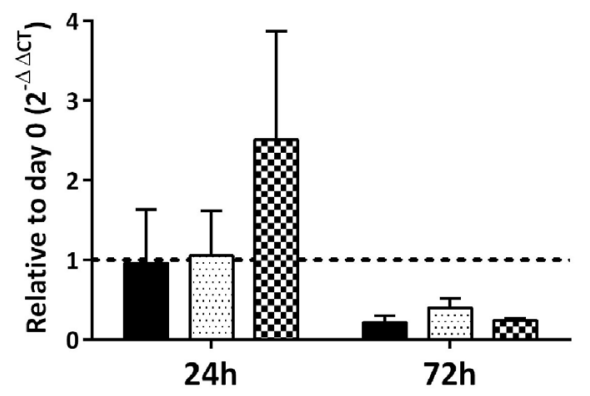

B

\section{COL2A1}

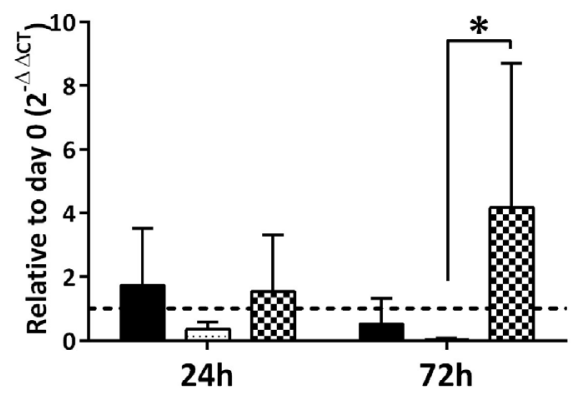

D

COL2A1/COL1A1

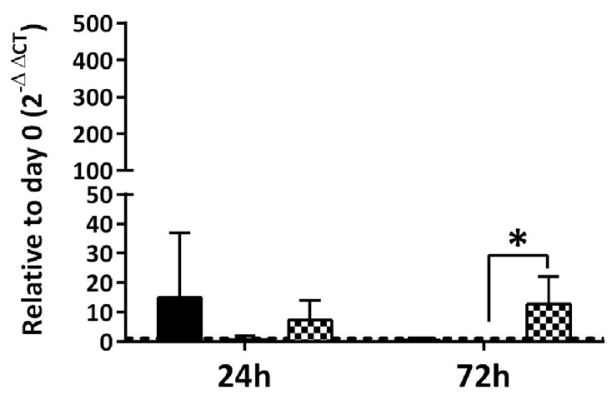

$\square$ FCS $⿴$ HAS 8 PRP Normoxia $\left(20 \% \mathrm{O}_{2}\right)$

E

MMP13

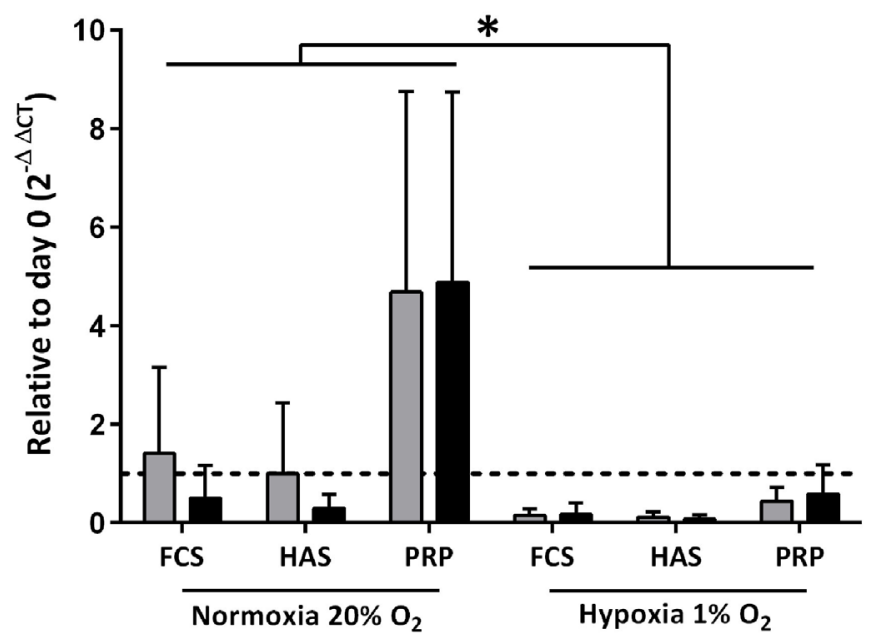

FIGURE 3 | Differences in relative expression of chondrogenic markers COL1A1, COL2A1, MMP3 (A-C), and the differentiation index (D) depicted to day 0 control under normoxic $\left(20 \% \mathrm{O}_{2}\right.$ ) conditions. Matrix metalloproteinase 13 (MMP13) expression significantly downregulated under hypoxia (1\% $\left.\mathrm{O}_{2}\right)$ than in normoxia (20\% $\left.\mathrm{O}_{2}\right)$ (E) as determined by reverse transcriptase quantitative real-time PCR of OA chondrocytes cultured in FCS, hyperacute serum (HAS), or platelet-rich plasma (PRP). Significant difference at * $p<0.05 ; n=3$ biological replicates.

similar decreases to the ones observed in normoxia (Figure S1 in Supplementary Material). This finding indicated that among the treatment conditions $10 \%$ PRP was capable of redifferentiating the dedifferentiated chondrocytes from day 0 to $72 \mathrm{~h}$ culture period (Figure 3A), while 10\% FCS or 10\% HAS led to further dedifferentiation (Figure 3B). No significant differences were observed in MMP3 under all conditions in both normoxia (Figure 3C) and hypoxia. MMP13 was downregulated from 24 to 
$72 \mathrm{~h}$ in $10 \% \mathrm{FCS}$ and $10 \%$ HAS under both normoxic and hypoxic conditions. In 10\% PRP, MMP13 was four times higher at both 24 and $72 \mathrm{~h}$ compared to day 0 under normoxia but downregulated under hypoxia (Figure 3E).

\section{DISCUSSION}

Our objective was to estimate the differences in growth factor levels among human PRP and HAS isolated from individual blood donors at different time points and examine their effect on chondrocyte proliferation in monolayers. We also examined the redifferentiation potential of dedifferentiated osteoarthritic chondrocytes. Our results suggest that growth factor levels do not differ among donors when isolated between any two of the pre-defined different isolation time points. HAS showed increases in chondrocyte proliferation versus PRP or FCS at the cost of dedifferentiation, but PRP has the potential to regenerate dedifferentiated chondrocytes on $2 \mathrm{D}$ substrates.

The PRP preparation used in our study contained six times more platelets than pre-prepared samples. PDGF-BB and LEPTIN were also found in higher amounts than bFGF or granulocyte colony-stimulating factor (GM-CSF) in PRP. These growth factor levels evaluated in our study have been studied for their underlying effect on chondrocytes in other studies. For example, GM-CSF has been reported to increase proliferation and anabolism in chondrocytes by inhibiting the IL-1 $\beta$-induced catabolic effect (Quintero et al., 2008; Marmotti et al., 2013). GM-CSF has also been shown to induce proteoglycan, aggrecan, and collagen synthesis of articular chondrocytes (Quintero et al., 2001). PDGF-BB has also been able to enhance chondrocyte proliferation through the extracellular signal-regulated kinase $1 / 2$ pathway (Xiao et al., 2014) and is active inhibitors of the IL-1 $\beta$ mediated activation of NF-kB and apoptosis in chondrocytes (Montaseri et al., 2011). bFGF is believed to be a key regulator of maintaining the chondrocyte phenotype during expansion. bFGF has a catabolic effect on dedifferentiation by upregulating the matrix metalloproteinases MMP1, MMP13 and downregulating aggrecan, collagen II in human OAC (Nummenmaa et al., 2015). Treatment of chondrocytes with the adipokine leptin can induce anabolic effects such as proliferation, differentiation, and cytoskeletal remodeling (Liang et al., 2011) and could lead to a pro-inflammatory stimulus in interaction with IL- $1 \beta$-induced nitric oxide (NO) production. This further leads to loss of the differentiated phenotype, apoptosis, and degradation of the extracellular matrix (Kim et al., 2002; Otero et al., 2005). Based on the data mentioned earlier, we quantified these factors and hypothesized that PRP or HAS could stimulate proliferation and differentiation of dedifferentiated OAC.

Obtaining adequate cell numbers for ACI treatment of cartilage defects is achieved by in vitro expansion on $2 \mathrm{D}$ surfaces. During this expansion, chondrocytes lose the characteristic round morphology, and phenotypic change to fibroblasts occurs as part of dedifferentiation. We found that the effects of HAS on increased proliferation in comparison to PRP or FCS were consistently higher from six blood donors on three osteoarthritic chondrocyte donors. Accelerating sufficient cell numbers in short culture time could reduce the waiting time of transplantation for
ACI procedures. However, such accelerated proliferation did not redifferentiate the chondrocytes when cultured with HAS rather more dedifferentiation accompanied by an increase in COL1A1 expression was observed. PRP did enhance proliferation on monolayer culture and at the same time induces redifferentiation by a decrease in COL1A1 and increased COL2A1 expression both under normoxic and hypoxic conditions. Spreafico et al. (2009) observed similar effects on increased anabolic gene expression, proliferation, and proteoglycan production when chondrocytes were cultured with PRP in comparison to human serum or FCS. Contrary to our study, some reports on PRP has been shown to have a detrimental effect on differentiation (Gaissmaier et al., 2005; Drengk et al., 2009) while others have reported a positive effect on differentiation (Akeda et al., 2006; Filardo et al., 2012). It may be that increased proliferation of cells leads to a decrease in type II collagen mRNA or vice versa in these studies as a cell cannot proliferate and differentiate at the same time. Recently, PRP's effect on adipose-derived stem cells toward chondrogenesis has been positively shown to induce chondrogenic differentiation without addition of exogenous growth factors and differentiated cells secreting less angiogenic/inflammatory markers (Pakfar et al., 2017). A similar effect was observed when chondrocyte/ MSC cocultures were cultured in the presence of PRP also by reducing the hypertrophic marker expression in cocultures (Ramezanifard et al., 2017). Several cytokines and signaling pathways involved in modulating chondrocyte dedifferentiation are reported as implications for cell-based cartilage therapies (Duan et al., 2015), and future interrogations should decipher and standardize these factors from PRP, HAS, and HS formulations.

Hypoxia was used to mimic the native environment in the current study, and we observed that low oxygen tension could inhibit the catabolic MMP13 expression during the redifferentiation process than in normoxia. Bouaziz et al. (2016) have shown that hypoxia-inducible factor $\alpha$ acts as a negative regulator of the Wnt $/ \beta$-catenin signaling which in turn downregulates MMP13 expression and as a result reduced cartilage loss occurs. Although PRP formulations vary disparately, one of the important factors to be noted is the leukocyte-rich or leukocyte-poor PRP. The presence of high leukocyte content increased the inflammatory factors such as IL-1 $\beta$ and catabolic cytokines (Sundman et al., 2011) whereas low leukocyte content led to dedifferentiation (Gaissmaier et al., 2005). Taking these factors into account, overcoming dedifferentiation on $2 \mathrm{D}$ surfaces than accelerated ex vivo expansion should be of consideration for cell-based therapies such as ACI and MACI. ACI is, however, not recommended by the International Cartilage Repair Society as a therapeutic option for patients over 40-50 years or those who suffer from osteoarthritis. However, a recent follow-up clinical study investigating ACI for young patients (38.3 years) suffering from early stage of osteoarthritis has indicated this technique to be a potential treatment option for degenerative disorders (Minas et al., 2010).

\section{CONCLUSION}

Our data indicate that HAS has its main effect on proliferation rather than redifferentiation while PRP enhances both proliferation and redifferentiaion of OAC during ex vivo expansion. 
Due to its autologous source and low cost, PRP has significant advantages over other therapies utilizing recombinant growth factors. The benefit of harvesting PRP from an individual patient offers a personalized formulation of a bioactive ECM milieu for enhancing chondrocyte culture conditions. We propose that OAC can be cultured with autologous PRP supplementation in growth medium for priming the cells for chondrogenic differentiation. This concept could be used in cell expansion for ACI procedures as a future improvement to existing therapies. Disadvantages include variability in plasma preparation protocols, differences in patients at various times of blood draws. HAS, however, enhances proliferation of $\mathrm{OAC}$ at the expense of increased dedifferentiation.

\section{ETHICS STATEMENT}

This study was carried out in accordance with the recommendations of Land Niederösterreich with written informed consent from all subjects. All subjects gave written informed consent in accordance with the Declaration of Helsinki. The protocol was approved by the "Land Niederösterreich by approval no. GS4-EK-4/249-2013.”

\section{REFERENCES}

Akeda, K., An, H. S., Okuma, M., Attawia, M., Miyamoto, K., Thonar, E. J.-M. A., et al. (2006). Platelet-rich plasma stimulates porcine articular chondrocyte proliferation and matrix biosynthesis. Osteoarthr. Cartil. 14, 1272-1280. doi:10.1016/j.joca.2006.05.008

Bouaziz, W., Sigaux, J., Modrowski, D., Devignes, C.-S., Funck-Brentano, T., Richette, P., et al. (2016). Interaction of HIF1 $\alpha$ and $\beta$-catenin inhibits matrix metalloproteinase 13 expression and prevents cartilage damage in mice. Proc. Natl. Acad. Sci. U.S.A. 113, 5453-5458. doi:10.1073/pnas.1514854113

Brandl, A., Angele, P., Roll, C., Prantl, L., Kujat, R., and Kinner, B. (2010). Influence of the growth factors PDGF-BB, TGF-betal and bFGF on the replicative aging of human articular chondrocytes during in vitro expansion. J. Orthop. Res. 28, 354-360. doi:10.1002/jor.21007

Dohan, D. M., Choukroun, J., Diss, A., Dohan, S. L., Dohan, A. J. J., Mouhyi, J., et al. (2006). Platelet-rich fibrin (PRF): a second-generation platelet concentrate. Part II: platelet-related biologic features. Oral Surg. Oral Med. Oral Pathol. Oral Radiol. Endod. 101, e45-e50. doi:10.1016/j.tripleo.2005.07.009

Drengk, A., Zapf, A., Stürmer, E. K., Stürmer, K. M., and Frosch, K. H. (2009). Influence of platelet-rich plasma on chondrogenic differentiation and proliferation of chondrocytes and mesenchymal stem cells. Cells Tissues Organs 189, 317-326. doi:10.1159/000151290

Duan, L., Ma, B., Liang, Y., Chen, J., Zhu, W., Li, M., et al. (2015). Cytokine networking of chondrocyte dedifferentiation in vitro and its implications for cell-based cartilage therapy. Am. J. Transl. Res. 7, 194-208.

Duval, E., Leclercq, S., Elissalde, J.-M., Demoor, M., Galéra, P., and Boumédiene, K. (2009). Hypoxia-inducible factor $1 \alpha$ inhibits the fibroblast-like markers type I and type III collagen during hypoxia-induced chondrocyte redifferentiation: hypoxia not only induces type II collagen and aggrecan, but it also inhibits type I and type III collagen in the hypoxia-inducible factor 1alpha-dependent redifferentiation of chondrocytes. Arthritis Rheum. 60, 3038-3048. doi:10.1002/ art. 24851

Egli, R. J., Bastian, J. D., Ganz, R., Hofstetter, W., and Leunig, M. (2008). Hypoxic expansion promotes the chondrogenic potential of articular chondrocytes. J. Orthop. Res. 26, 977-985. doi:10.1002/jor.20603

Filardo, G., Di Matteo, B., Di Martino, A., Merli, M. L., Cenacchi, A., Fornasari, P., et al. (2015). Platelet-rich plasma intra-articular knee injections show no superiority versus viscosupplementation: a randomized controlled trial. Am. J. Sports Med. 43, 1575-1582. doi:10.1177/0363546515582027

Filardo, G., Kon, E., Di Martino, A., Di Matteo, B., Merli, M. L., Cenacchi, A., et al. (2012). Platelet-rich plasma vs hyaluronic acid to treat knee degenerative

\section{AUTHOR CONTRIBUTIONS}

SN acquired funding for this research. VJ, EN-M, and ZL conceived idea, designed experiments, analyzed data, and interpreted results. VJ, EN-M, and CB performed experiments. VJ, ZL, and $\mathrm{SN}$ drafted the manuscript. All authors reviewed, revised, and approved the final version of the manuscript.

\section{FUNDING}

The authors gratefully acknowledge the financial support of the NÖ Forschungs- und Bildungsges.m.b.H. (NFB) and the provincial government of Lower Austria through the Life Science Calls (Project ID: LS12-001).

\section{SUPPLEMENTARY MATERIAL}

The Supplementary Material for this article can be found online at http://www.frontiersin.org/article/10.3389/fbioe.2017.00075/ full\#supplementary-material.

pathology: study design and preliminary results of a randomized controlled trial. BMC Musculoskelet. Disord. 13:229. doi:10.1186/1471-2474-13-229

Gaissmaier, C., Fritz, J., Krackhardt, T., Flesch, I., Aicher, W. K., and Ashammakhi, N. (2005). Effect of human platelet supernatant on proliferation and matrix synthesis of human articular chondrocytes in monolayer and three-dimensional alginate cultures. Biomaterials 26, 1953-1960. doi:10.1016/j.biomaterials. 2004.06.031

Henderson, J. H., Ginley, N. M., Caplan, A. I., Niyibizi, C., and Dennis, J. E. (2010). Low oxygen tension during incubation periods of chondrocyte expansion is sufficient to enhance postexpansion chondrogenesis. Tissue Eng. Part A 16, 1585-1593. doi:10.1089/ten.tea.2009.0411

Heywood, H. K., and Lee, D. A. (2010). Low oxygen reduces the modulation to an oxidative phenotype in monolayer-expanded chondrocytes. J. Cell. Physiol. 222, 248-253. doi:10.1002/jcp.21946

Jeyakumar, V., Halbwirth, F., Niculescu-Morzsa, E., Bauer, C., Zwickl, H., Kern, D., et al. (2016). Chondrogenic gene expression differences between chondrocytes from osteoarthritic and non-OA trauma joints in a 3D collagen type I hydrogel. Cartilage 8, 191-198. doi:10.1177/1947603516657641

Kang, S.-W., Yoo, S. P., and Kim, B.-S. (2007). Effect of chondrocyte passage number on histological aspects of tissue-engineered cartilage. Biomed. Mater. Eng. 17, 269-276.

Kawanishi, M., Oura, A., Furukawa, K., Fukubayashi, T., Nakamura, K., Tateishi, T., et al. (2007). Redifferentiation of dedifferentiated bovine articular chondrocytes enhanced by cyclic hydrostatic pressure under a gas-controlled system. Tissue Eng. 13, 957-964. doi:10.1089/ten.2006.0176

Kim, S. J., Ju, N. W., Oh, C. D., Yoon, Y. M., Song, W. K., Kim, J. H., et al. (2002) ERK-1/2 and p38 kinase oppositely regulate nitric oxide-induced apoptosis of chondrocytes in association with p53, caspase-3, and differentiation status. J. Biol. Chem. 277, 1332-1339. doi:10.1074/jbc.M107231200

Liang, J., Feng, J., Wu, W. K. K., Xiao, J., Wu, Z., Han, D., et al. (2011). Leptinmediated cytoskeletal remodeling in chondrocytes occurs via the RhoA/ROCK pathway. J. Orthop. Res. 29, 369-374. doi:10.1002/jor.21257

Livak, K. J., and Schmittgen, T. D. (2001). Analysis of relative gene expression data using real-time quantitative PCR and the $2^{\wedge}(-\Delta \Delta \mathrm{CT})$ method. Methods 25 , 402-408. doi:10.1006/meth.2001.1262

Markway, B. D., Cho, H., and Johnstone, B. (2013). Hypoxia promotes redifferentiation and suppresses markers of hypertrophy and degeneration in both healthy and osteoarthritic chondrocytes. Arthritis Res. Ther. 15, R92. doi:10.1186/ $\operatorname{ar} 4272$

Marmotti, A., Bonasia, D. E., Bruzzone, M., Rossi, R., Castoldi, F., Collo, G., et al. (2013). Human cartilage fragments in a composite scaffold for single-stage 
cartilage repair: an in vitro study of the chondrocyte migration and the influence of TGF- $\beta 1$ and G-CSF. Knee Surg. Sports Traumatol. Arthrosc. 21, 1819-1833. doi:10.1007/s00167-012-2244-7

Martin, I., Vunjak-Novakovic, G., Yang, J., Langer, R., and Freed, L. E. (1999). Mammalian chondrocytes expanded in the presence of fibroblast growth factor 2 maintain the ability to differentiate and regenerate three-dimensional cartilaginous tissue. Exp. Cell Res. 253, 681-688. doi:10.1006/excr.1999.4708

Mesenchymal, M. (2009). Influence of three-dimensional hyaluronic acid stem cell chondrogenesis. Gene Expr. 15, 243-254. doi:10.1089/ten.tea.2008.0067

Minas, T. (2001). Autologous chondrocyte implantation for focal chondral defects of theknee.Clin. Orthop. Relat. Res. (391Suppl):S349-S361.doi:10.1097/00003086200110001-00032

Minas, T., Gomoll, A. H., Solhpour, S., Rosenberger, R., Probst, C., and Bryant, T. (2010). Autologous chondrocyte implantation for joint preservation in patients with early osteoarthritis. Clin. Orthop. Relat. Res. 468, 147-157. doi:10.1007/ s11999-009-0998-0

Montaseri, A., Busch, F., Mobasheri, A., Buhrmann, C., Aldinger, C., Rad, J. S., et al. (2011). IGF-1 and PDGF-bb suppress IL-1 1 -induced cartilage degradation through down-regulation of NF-kB signaling: involvement of Src/PI-3k/AKT pathway. PLoS ONE 6:e28663. doi:10.1371/journal.pone.0028663

Nehrer, S., Breinan, H. A., Ramappa, A., Young, G., Shortkroff, S., Louie, L. K., et al. (1997). Matrix collagen type and pore size influence behaviour of seeded canine chondrocytes. Biomaterials 18, 769-776. doi:10.1016/S0142-9612 (97)00001-X

Nehrer, S., Chiari, C., Domayer, S., Barkay, H., and Yayon, A. (2008). Results of chondrocyte implantation with a fibrin-hyaluronan matrix: a preliminary study. Clin. Orthop. Relat. Res. 466, 1849-1855. doi:10.1007/s11999-008-0322-4

Niemeyer, P., Steinwachs, M., Erggelet, C., Kreuz, P. C., Kraft, N., Kostler, W., et al. (2008). Autologous chondrocyte implantation for the treatment of retropatellar cartilage defects: clinical results referred to defect localisation. Arch. Orthop. Trauma Surg. 128, 1223-1231. doi:10.1007/s00402-007-0413-9

Nummenmaa, E., Hämäläinen, M., Moilanen, T., Vuolteenaho, K., and Moilanen, E. (2015). Effects of FGF-2 and FGF receptor antagonists on MMP enzymes, aggrecan, and type II collagen in primary human OA chondrocytes. Scand. J. Rheumatol. 44, 321-330. doi:10.3109/03009742.2014.1000372

Otero, M., Lago, R., Lago, F., Reino, J. J. G., and Gualillo, O. (2005). Signalling pathway involved in nitric oxide synthase type II activation in chondrocytes: synergistic effect of leptin with interleukin-1. Arthritis Res. Ther. 7, R581-R591. doi:10.1186/ar1708

Pakfar, A., Irani, S., and Hanaee-Ahvaz, H. (2017). Expressions of pathologic markers in PRP based chondrogenic differentiation of human adipose derived stem cells. Tissue Cell 49, 122-130. doi:10.1016/j.tice.2016.11.001

Pei, M., Seidel, J., Vunjak-Novakovic, G., and Freed, L. E. (2002). Growth factors for sequential cellular de- and re-differentiation in tissue engineering. Biochem. Biophys. Res. Commun. 294, 149-154. doi:10.1016/S0006-291X(02)00439-4

Peterson, L., Minas, T., Brittberg, M., Nilsson, A., Sjögren-Jansson, E., and Lindahl, A. (2000). Two- to 9-year outcome after autologous chondrocyte transplantation of the knee. Clin. Orthop. Relat. Res. (374):212-234. doi:10.1097/00003086200005000-00020

Quintero, M., Colantuoni, G., Khatib, A. M., Panasyuk, A., Lomri, A., and Mitrovic, D. R. (2001). Granulocyte-macrophage colony stimulating factor activates proteoglycan, type II collagen, and cAMP production by rat articular chondrocytes through specific binding sites. J. Rheumatol. 28, 2075-2084.

Quintero, M., Riera, H., Colantuoni, G., Khatib, A. M., Attalah, H., Moldovan, F., et al. (2008). Granulocyte-macrophage colony stimulating factor is anabolic and interleukin-1beta is catabolic for rat articular chondrocytes. Cytokine 44, 366-372. doi:10.1016/j.cyto.2008.10.003

Ramezanifard, R., Kabiri, M., and Hanaee-Ahvaz, H. (2017). Effects of platelet rich plasma and chondrocyte co-culture on MSC chondrogenesis, hypertrophy and pathological responses. EXCLI J. 16, 1031-1045. doi:10.17179/excli2017-453

Schnabel, M., Marlovits, S., Eckhoff, G., Fichtel, I., Gotzen, L., Vécsei, V., et al. (2002). Dedifferentiation-associated changes in morphology and gene expression in primary human articular chondrocytes in cell culture. Osteoarthr. Cartil. 10, 62-70. doi:10.1053/joca.2001.0482

Smith, P. A. (2016). Intra-articular autologous conditioned plasma injections provide safe and efficacious treatment for knee osteoarthritis: an FDA-sanctioned, randomized, double-blind, placebo-controlled clinical trial. Am. J. Sports Med. 44, 884-891. doi:10.1177/0363546515624678

Spreafico, A., Chellini, F., Frediani, B., Bernardini, G., Niccolini, S., Serchi, T., et al. (2009). Biochemical investigation of the effects of human platelet releasates on human articular chondrocytes. J. Cell. Biochem. 108, 1153-1165. doi:10.1002/ jcb. 22344

Stevens, M. M., Qanadilo, H. F., Langer, R., and Shastri, V. P. (2004). A rapid-curing alginate gel system: utility in periosteum-derived cartilage tissue engineering. Biomaterials 25, 887-894. doi:10.1016/j.biomaterials.2003.07.002

Stewart, M. C., Saunders, K. M., Burton-Wurster, N., and Macleod, J. N. (2000). Phenotypic stability of articular chondrocytes in vitro: the effects of culture models, bone morphogenetic protein 2, and serum supplementation. J. Bone Miner. Res. 15, 166-174. doi:10.1359/jbmr.2000.15.1.166

Sundman, E. A., Cole, B. J., and Fortier, L. A. (2011). Growth factor and catabolic cytokine concentrations are influenced by the cellular composition of platelet-rich plasma. Am. J. Sports Med. 39, 2135-2140. doi:10.1177/0363546511417792

Vonk, L. A., de Windt, T. S., Kragten, A. H. M., Beekhuizen, M., Mastbergen, S. C., Dhert, W. J. A., et al. (2014). Enhanced cell-induced articular cartilage regeneration by chondrons; the influence of joint damage and harvest site. Osteoarthr. Cartil. 22, 1910-1917. doi:10.1016/j.joca.2014.08.005

Xiao, J., Chen, X., Xu, L., Zhang, Y., Yin, Q., and Wang, F. (2014). PDGF regulates chondrocyte proliferation through activation of the GIT1- and PLC $\gamma 1$-mediated ERK1/2 signaling pathway. Mol. Med. Rep. 10, 2409-2414. doi:10.3892/ mmr.2014.2506

Conflict of Interest Statement: The authors declare that the research was conducted in the absence of any commercial or financial relationships that could be construed as a potential conflict of interest.

The reviewer CG and handling editor declared their shared affiliation.

Copyright $\odot 2017$ Jeyakumar, Niculescu-Morzsa, Bauer, Lacza and Nehrer. This is an open-access article distributed under the terms of the Creative Commons Attribution License (CC BY). The use, distribution or reproduction in other forums is permitted, provided the original author(s) or licensor are credited and that the original publication in this journal is cited, in accordance with accepted academic practice. No use, distribution or reproduction is permitted which does not comply with these terms. 